\title{
Optimal Models of Home Energy Management System
}

\author{
Qingzhu Wan ${ }^{\mathrm{a}}$, Yalan Chen ${ }^{\mathrm{b}}$ and Yuan Bian ${ }^{\mathrm{c}}$ \\ North China University of Technology, Beijing 100144, China \\ a13501009879@163.com, bchenyalanjane@163.com, c1191796395@qq.com
}

Keywords: home energy management system, optimal model, load rate, genetic algorithm.

\begin{abstract}
Residential sector is a potential field of energy conservation and emission reduction in smart grid. Smart home can reduce electricity cost and carbon emissions by optimal the energy use of household appliances. We bulid day-ahead dispatch schedule models which are minimize electricity cost model and minimize carbon emissions model. In the models, we both combined with the goal of electricity safety. We improved Genetic Algorithm to solve our models. The simulation results show that the models can effectively and safely optimize the home energy management.
\end{abstract}

\section{Introduction}

Electrical demand has been dramatically increased all over the world in recent years. Residential electricity consumption account for $36.6 \%$ of total power consumption ${ }^{[1]}$. With real-time pricing carrying out, home energy management becomes more and more important in our society. As the reason of many loads in home are neither controllable nor measurable, the traditional management system only can scheduled generation resources ${ }^{[2][3]}$. However, new technologies has been deployed including smart meters, controllable appliances, and communication capabilities. It gives a chance to household appliances participate in the energy management.

Home Energy Management System (HEMS) is a intelligent management system. HEMS can realize the function of online control under the power company's protocol and the user's preference ${ }^{[4]}$. It provides a lot of benefits such as saving electricity cost for consumers, reducing peak demand for grid, meeting the demand side requirements and decreasing the carbon emissions.

The main research area in HEMS is optimal dispatch strategy. Recent researchs have a number of achievements in this area. In literature [4-6], they classified the household appliances in the similar way, bulit up models with different goals, and solved it respectively. They used different ways to enhance electricity efficiency of home. In those researchs, the main factors include: maximum renewable energy utilization, maximize consumer's satisfaction, minimize consumer's cost, and minimize carbon emissions. However, one of the energy management basis is electricity safety. It's irresponsible to take as much household appliances as possible into the scheduling without considering electricity safety. The lines maybe overload when the result of optimal dispatch model make overmuch appliances in the same line work at the same time. In brief, safety is the primary part of the HEMS.

In this paper, we propose a HEMS optimal dispatch strategy taking account of electricity safety and explore its optimal algorithm. For this purpose, we using electricity safety as a goal separately combined with electricity cost and carbon emissions set up two models which are minimize electricity cost model and minimize carbon emissions model. We improved Genetic Algorithm (GA) to get the optimal result of those models. The simulation at the end of this paper has proved that the optimal dispatch strategy can make users live in a money-saving or environment-friendly way while keeping electricity safety.

\section{Structure of HEMS}

HEMS contains distributed generation, energy storage system, and household appliance. The energy storage system is used for improve renewable energy utilization. However, it not only increaces the cost of system, but also hidden security risks when the storage is in charging or 
discharging. Furthermore, the improper disposal may cause environmental pollution ${ }^{[7]}$. Thus, we design a HEMS without energy storage. The topological structure of HEMS as shown in Fig.1.

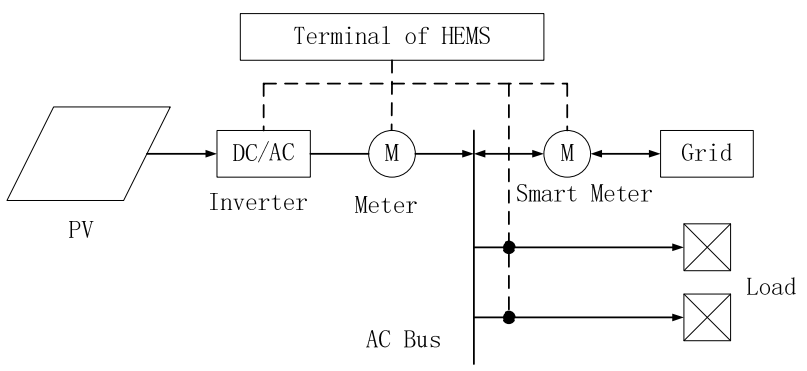

Fig. 1 Topological structure of home energy management system

The house is equipped with a rooftop photovoltaic (PV) system, a smart meter and a set of household appliances. To date, the PV system has three patterns: all energy self consumption, all energy sell to utility grid, and sell to utility grid when there is spare energy after self consumption. In this system, we choice the third pattern which is more flexible in using electricity. The system has two meters, one is an ordinary meter which used to measure energy from PV, and the other is a smart meter which can measure bilateral power flow between the house and utility grid. All of those are monitored by the terminal of HEMS to realize the optimal dispatch results normal running.

\section{Optimal Dispatch Strategy of HEMS}

\subsection{Object of Optimal Dispatch}

The objects of optimal dispatch are the switch of electricity transaction and household appliances. The variates are those objects' working condition in every period. The working condition only can be on or off. The 1 stand for on and 0 stand for off. Peculiarly, we regard the switch of electricity transaction 1 as the house sell electricity and regard 0 as the house buy electricity. The household appliances are divided into must run loads and schedulable loads. The must run loads such as refrigerator and lightings which have a great influence in the user's life will just be a basis of dispatch. The schedulable loads include interrupted loads, non-interrupted loads and thermostatically controlled loads. The distinction of the interrupted loads and the non-interrupted loads is whether their operations can be interrupted once they have been started. The thermostatically controlled loads such as air condition or water heater. The working condition of thermostatically controlled loads is decided by the temperature.

Compared with other classifications, this classification is more comprehensive about household appliances. In this classification, the must run loads can be forecast through the history data of the household appliances use electricity. The interrupted loads and the non-interrupted loads are set working time range $[\mathrm{a}, \mathrm{b}]$, working periods $\mathrm{N}$ and the shortest contiguous working periods $\mathrm{L}$ by the user. The non-interrupted loads' shortest contiguous working periods $\mathrm{L}$ is equal to its working periods N. The thermostatically controlled loads are set each period temperature demand by the user.

\subsection{Dispatch Strategy}

We divide a day into 24 periods averagely. The dispatch strategy is that we put the appliances runing time into appropriate periods to achieve the goal which we set. In detail, the house gets the next day real-time pricing from the electricity company. The house get the next day weather forecast information from meteorological service website, and then forecasts energy produced by PV system through this information. In general, the energy produced by PV system at noon is more than the energy produced at night and the electricity from utility grid in peak is more expensive than it in valley. In order to lower costs, the strategy is to arrange appliances working in the periods which is price valley. In order to lower carbon emissions, the strategy is to arrange appliances working at the periods when the PV system is active.

First, HEMS gathers the real-time pricing, the forecasting data of PV system and the must run loads electricity consumption. Second, user set the schedulable loads running requirements through 
human-computer interface. Third, solve the model according to these data and constraints. Then, the user gets a scheduling about appliances running condition and electricity transaction condition in every period of the next day. Finally, the terminal of HEMS will controll the switch of electricity transaction and all the appliances to work in the next day according to the scheduling which is confirmed by the user.

\section{Optimal Dispatch Models of HEMS}

In this paper, we build three objective functions about electricity cost, carbon emissions and electricity safety. For most of people, they care electricity cost more than carbon emissions. But environmentalist is just opposite. However, whether ordinary people or environmentalists, they all care about electricity safety. Therefore, we combine electricity cost with electricity safety as the minimize electricity cost model; we combine carbon emissions with electricity safety as the minimize carbon emissions model. Users could make a choice from the two models based on their preferences.

\subsection{Electricity Cost Objective Function}

The cost mainly produced in three aspects: buy electricity from electricity company, sell electricity to electricity company and state subsidize for PV. Therefore, the electricity cost objective function consideres these aspects.

$$
\begin{gathered}
\min \text { Cost }=\sum_{t=1}^{24}\left[C_{1}(t)-C_{2}(t)\right] \\
C_{1}(t)= \begin{cases}P_{b}(t) \times P(t) \times T ; & P(t)>0 \\
P_{s}(t) \times P(t) \times T ; & P(t) \leq 0\end{cases} \\
C_{2}(t)=G(t) \times P_{G}
\end{gathered}
$$

where Cost denote the household energy cost for a single day; $\mathrm{T}$ is time duration of a period, $\mathrm{T}=1 \mathrm{~h}$; $\mathrm{t}$ is the serial number of $\mathrm{T} ; \mathrm{C}_{1}(\mathrm{t})$ denote the cost of electricity trade between the house and electricity company at NO.t period; $\mathrm{C}_{2}(\mathrm{t})$ denote the state subsidy at NO.t period; $\mathrm{P}_{\mathrm{b}}(\mathrm{t})$ is the price that the house buy electricity at NO.t period; $P_{s}(t)$ is the price that the house sell electricity at NO.t period; $G(t)$ is the PV generation at NO.t period; $\mathrm{PG}$ is the state subsidy price of $\mathrm{PV}$ generation; $\mathrm{P}(\mathrm{t})$ is the power that the house trades with electricity company at NO.t period, when $\mathrm{P}(\mathrm{t})>0$, it means the house buys electricity at NO.t period, when $\mathrm{P}(\mathrm{t})<0$, it means the house sells electricity at NO.t period.

\subsection{Carbon emissions Objective Function}

In recent years, global warming becoming more and more serious. Users hope to reduce carbon emissions. Meanwhile, the way of appliances working should not reduce the user's comfort. The main composition of utility grid electricity is traditional coal generation which cause carbon emissions. The more PV energy used, the less utility grid energy used, the more cleaner house energy use. Thus, the carbon emissions objective function is expressed as follow:

$$
\min F=\sum_{t \in \Omega} \delta \times P(t) \times T
$$

where $\mathrm{F}$ denote the whole day carbon emissions of the house; $\delta$ represents the carbon emission per kilowatt-hour; $\Omega$ is a set which constitute all the periods of $\mathrm{P}(\mathrm{t})>0$.

\subsection{Electricity Safety Objective Function}

We consider the house safety is about load rate of each line. The household circuit system diagram is shown in Fig.2. 


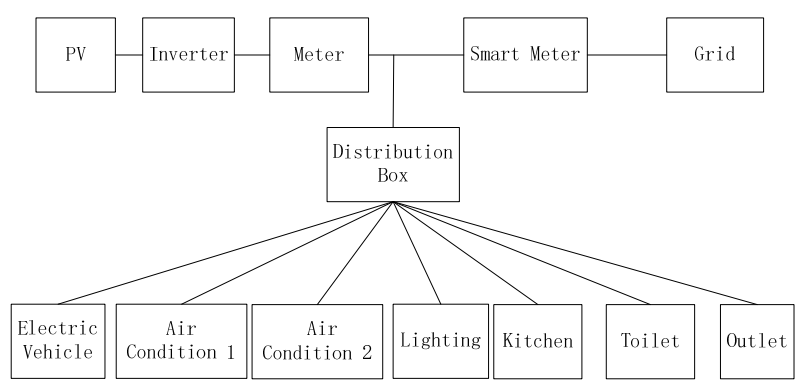

Fig. 2 household circuit system diagram

There are seven lines from distribution box to appliances. We use i represent it and numbered it from 1 to 7 . The active power flow of each line is expressed as follow:

$$
P_{l i}(t)=M_{i}(t)+\sum_{j} W_{i j} \times A_{i j}(t)
$$

where, $\mathrm{M}_{\mathrm{i}}(\mathrm{t})$ is the power demand of must run loads in NO.i line; $\mathrm{W}_{\mathrm{ij}}$ is the power demand of NO.j appliance on NO.i line; $\mathrm{A}_{\mathrm{ij}}(\mathrm{t})$ stands for the working condition of NO.j appliance on NO.i line at NO.t period. $A_{i j}(t)=1$ means this appliance is working at NO.t period, $A_{i j}(t)=0$ means the opposite.

The load rate of each line ${ }^{[8]}$ is expressed as follow:

$$
R_{i}(t)=\frac{P_{l i}(t)}{P_{l i \max }}
$$

where, $\mathrm{P}_{\text {limax }}$ is the power rating of NO.i line.

In theory, the lower $R_{i}(t)$ is, the safer users are. However, the goal is to ensure electricity safety instead of prevent users from using electricity. It doesn't need the objective function to be minimum, but to keep in a safe range.

The two models which we built are multi-objective optimization model. We adopt the main goal method to convert the safety objective function into a constraint. The multi-objective optimization models turn into single-objective optimization models.

\subsection{Constraint}

1)Safety constraint which is convert from safety objective function:

$$
R_{i}(t) \leq \alpha
$$

where $\alpha$ is a safety constant.

2)Power balance constraint:

$$
\sum_{i}\left(\sum_{j} W_{i j} \times A_{i j}(t)\right)+M_{i}(t)=G(t)+P(t)
$$

If $\mathrm{P}(\mathrm{t})>0, \mathrm{~S}(\mathrm{t})=0$; If $\mathrm{P}(\mathrm{t})<0, \mathrm{~S}(\mathrm{t})=1$. Where $\mathrm{S}(\mathrm{t})$ refers to the house whether sells electricity or not.

3)Appliances working periods constraint

$$
\sum_{t=1}^{24} A_{i j}(t)=N_{i j}
$$

where, $\mathrm{N}_{\mathrm{ij}}$ stands for the total working periods of NO.j appliance on NO.i line.

4)Constraint of the shortest contiguous working periods:

$$
A_{i j}(t+x)=1, \forall x \in\left[0, L_{i j}\right] \& B_{i j}(t)=1
$$

where, $\mathrm{L}_{\mathrm{ij}}$ is the shortest contiguous working periods of the NO.j appliance on NO.i line; $\mathrm{B}_{\mathrm{ij}}(\mathrm{t})$ means the appliance start working at NO.t period.

5)Constraint of thermostatically controlled loads:

The thermostatically controlled loads should keep the controlled temperature in a range which the user could accept.

The constraint of temperature:

$$
T=T_{s} \pm \Delta T
$$


where $\mathrm{T}$ is the actual temperature; $\mathrm{Ts}$ is the expected temperature set by the user; $\Delta \mathrm{T}$ is the temperature deviation.

6)The model of thermostatically controlled loads ${ }^{[6][9]}$ (taking air condition as an example ):

$$
\left.T_{\text {roon }}(t+1)=T_{\text {roon }}(t) e^{\frac{T}{R C}}+\left[T_{\text {air }}(t)-A_{i j}(t) Q R\right)\right]\left(1-e^{\frac{T}{R C}}\right)
$$

where Troom represents the indoor temperature; Tair represents the outdoor temperature; $\mathrm{R}$ stands for equivalent total thermal resistance; $\mathrm{C}$ stands for heat capacity; $\mathrm{Q}$ stands for equivalent heat rate; Aij(t) refers to the running state of air condition. Water heater has the similar model.

\section{Optimal Algorithm of HEMS}

We adopt binary $\mathrm{GA}^{[10]}$ (Genetic Algorithm) to solve our models because the models are 0-1 integer programming. In order to precaution GA missing optimal solution, we adopt Elitist Preserved Genetic Algorithm ${ }^{[11-12]}$. In GA, it is difficult to select a contiguous code that suit the requirement of contiguous working periods through crossover and mutation. Thus, each individual has been improved before calculating its fitness. In each new generation, the code of chromosome will be moved to right $1,2, \ldots\left(\mathrm{L}_{\mathrm{ij}}-1\right)$. Then, Add up all the new created chromosome and the original chromosome to get the chromosome which has the contiguous code. Thus, it is easier to select a suitable contiguous code. We employ penalty function to deal with other constraints. Fig. 3 shows the flow chart of the improved algorithm.

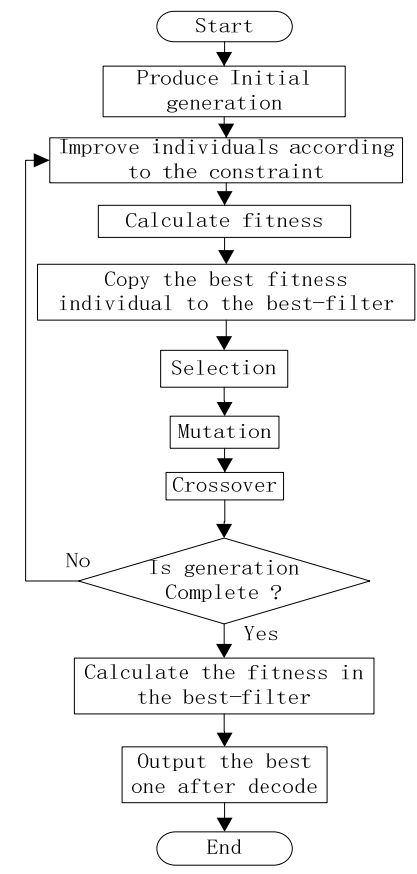

Fig. 3 Flow chart of the algorithm

\section{Simulation Results}

This case simulates the models combined electricity safety. The simulation results is shown in Fig.4 to Fig.6. The load rates are balance. The NO.5 line load rate of those models are 0.75 at 17:00-18:00. The load rate of each line keeps in a safety range as shown in Fig.5. It demonstrate that the safety goal reacts on the HEMS. 


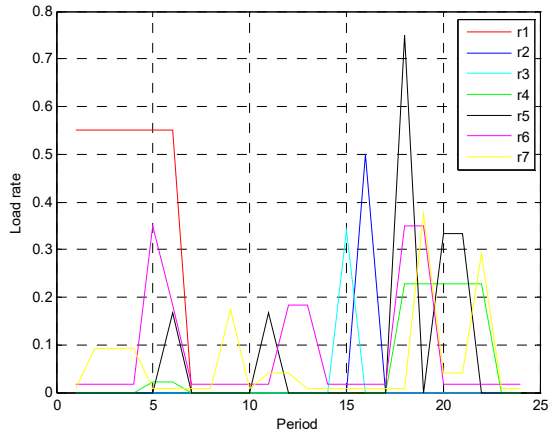

(a) minimize the electricity cost

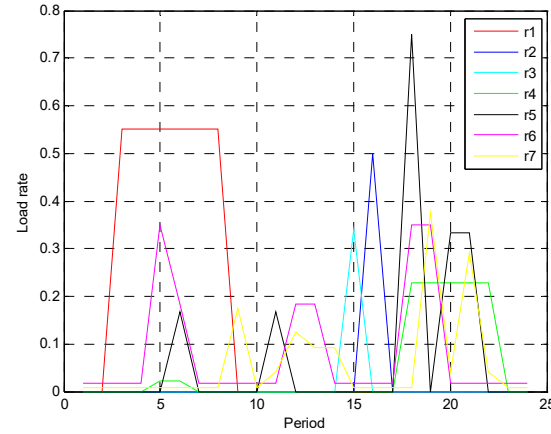

(b) minimize the carbon emissions

Fig. 4 Load rate of each line

Fig.5 is the result of the minimize electricity cost model. In order to lower the cost, the appliance $\mathrm{A}_{11}, \mathrm{~A}_{72}, \mathrm{~A}_{73}$ was moved to the night at 0:00-6:00, 22:00-23:00, 0:00-3:00, which has a lower price.

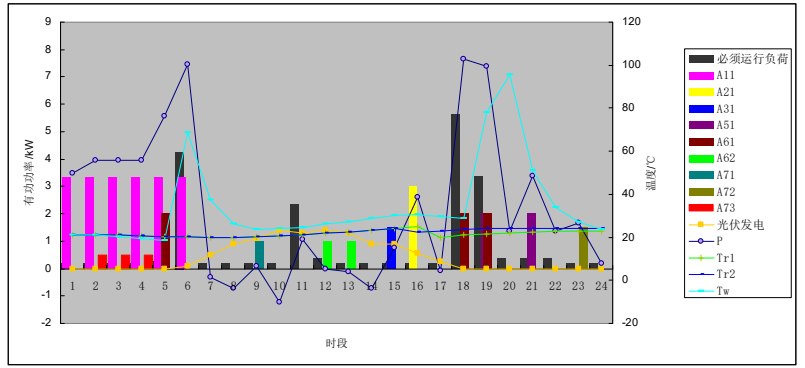

Fig. 5 Result of minimize the electricity cost model

Fig. 6 is the result of the minimize carbon emissions model. It took the advantage of the PV power to schedule the household appliances. The appliance $A_{11}, A_{62}, A_{73}$ was scheduled at 2:00-8:00, 12:00-14:00, 9:00-11:00, in which the PV system is active. The utilization rate of PV power was improved. The more PV power used, the less grid power used. Thus, the carbon emissions was reduced.

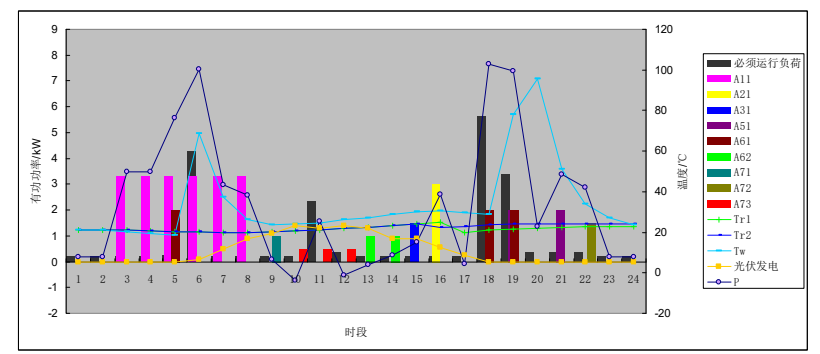

Fig. 6 Result of minimize the carbon emissions model

In those models, the thermostatically controlled loads work well. As shown in Fig.5 and Fig.6, the temperature of room 1 , room $2 \mathrm{kept}$ around $23^{\circ} \mathrm{C}$ and the temperature of water reached $48.5^{\circ} \mathrm{C}, 71.2^{\circ} \mathrm{C}$ respectively at 5:00-6:00, 18:00-20:00. Since we improved GA, it is clear to see that the results perfectly keep non-interrupted loads working continuously, such as appliance $\mathrm{A}_{11}, \mathrm{~A}_{62}, \mathrm{~A}_{73}$.

A comparison between unscheduled pattern and the two models in this paper is shown in Table 1.

Table 1 Comparison of results

\begin{tabular}{|c|c|c|c|}
\hline & \multirow{2}{*}{$\begin{array}{c}\text { Unused } \\
\text { optimizing }\end{array}$} & Minimize electricity cost & Minimize carbon emissions \\
\cline { 3 - 4 } & 25.19 & 19.15 & 21.64 \\
\hline $\begin{array}{c}\text { Cost } ¥ ¥ \\
\text { Carbon } \\
\text { emissions/kg }\end{array}$ & 59.47 & 57.56 & 55.71 \\
\hline
\end{tabular}

In the case of unused optimizing, the cost is $¥ 25.19$ and the carbon emissions is $59.47 \mathrm{~kg}$. The cost in the minimize electricity cost model is $¥ 19.15$. The cost reduced $23.98 \%$ in the minimize electricity cost model. The carbon emissions in the minimize the carbon emissions model is $55.71 \mathrm{~kg}$. The 
carbon emissions reduced $6.3 \%$ in the minimize carbon emissions model. These results indicate that the strategy we proposed can effectively solve the problems of HEMS in different goals.

From the table 1, we could find that the two models have different characteristic. The minimize electricity cost model is good at the cost saving, but not good at the carbon emissions reducing. The minimize carbon emissions model is just opposite. Therefore, the user could chose the differert model base on their own desire.

\section{Conclusions}

In this paper, we have presented a strategy of Home Energy Management System optimal dispatch. Based on this strategy, we set up two models. The main idea is to optimal the house energy use under the real-time pricing and PV power. The goal is to achieve minimize electricity cost or minimize carbon emissions, while keeping the house electricity safe. The study also propose an advanced GA to solve the models. Finally, through the simulated results, it proved that the models can effectively help the user to schedule energy use and eliminate the security risks.

\section{Acknowledgments}

This work is supported by the Education Committee of Beijing basic research program of China(XN097) and Foundation of Collaborative Innovation Center of Key Power Energy-Saving Technologies in Beijing (PXM2016_014212_000016).

\section{References}

[1] Zhang Yan-yu,Zeng Peng,Zang Chuan-zhi.Review of home energy management system in smart grid[J].Power System Protection and Control, 2014, 42(18):144-151.

[2] Pengwei Du, Ning Lu. “Appliance Commitment for Household Load Scheduling," IEEE Transactions on Smart Grid, vol. 2,pp.411-419Jun. 2011.

[3] Jidong Wang, Zhiqing Sun, Yue Zhou and Jiaqiang Dai.Optimal Dispatching Model of Smart Home Energy Management System[J]. IEEE PESISGT ASIA 2012.

[4] Zhang Yan,Zhang Tao,Liu Yajie,Guo Bo.Optimal Energy Management of a Residential Local Energy Network Based on Model Predictive Control[J]. Proceedings of the CSEE,2015,35(14): 3656-3666.

[5] Liu Jinghao,He Rong,Li Renfa,Zeng Gang.Optimal scheduling model for home energy management system based on real-time electricity pricing[J].Application Research of Computers,2015,32(1):132-137.

[6] Wang Shou xiang,Sun Zhi qing,Liu Zhe.Co-scheduling Strategy of Home Energy for Smart Power Utilization[J]. Automation of Electric Power Syetems,2015,39 (17):108-113.

[7] Li Lei, Yang Chun, Xie Xiaofeng. Current situation, opportunities and challenges of energy storage industry in China[J]. Chemical Industry and Engineering Progress, 2011，30:748-754.

[8] Sun Xin,Lv Yuechun,Gao Jun,Xia Qing.Power Grid Economy and Security Coordination Lean Method[J].Power System Technology, 2009,33(11):12-17.

[9] Dolan P S, Nehrir M H, Gerez V.Development of a Monte Carlo based aggregate model for residential electric water heater loads[J].Electric Power Systems Research, 1996,36(1):29-35.

[10] Pengfei Guo, Xuezhi Wang, Yingshi Han. The Enhanced Genetic Algorithms for the Optimization Design[C]. 2010 3rd International Conference on Biomedical Engineering and Informatics. 
[11] Long Duy Ha, Ploix S, Zamai E. Tabu search for the optimization of household energy consumption//Proc IEEE International Conference on Information Reuse and Integration. Waikoloa, Hawaii, USA: IEEE Press,2006: 86-92.

[12] Shen Jiajian, Yang Genke, Pan Changchun.Dynamic Model and Optimization of Hybrid Energy Management in Smart Home[J]. Microcomputer Applications,2012,28(4):9-14. 